\title{
A Trial of Using Solvent Extraction for Phosphorus Recovery
}

\author{
Onesmus N. Mwabonje ${ }^{1, *}$, Jia-Qian Jiang ${ }^{1,2}$ \\ ${ }^{1}$ CEHE, C5, Faculty of Engineering and Physical Sciences, University of Surrey, Guildford, Surrey, GU2 7XH, UK \\ ${ }^{2}$ School of the Built and Natural Environment, Glasgow Caledonian University, Cowcaddens Road, Glasgow, Scotland, \\ G4 OBA, UK \\ *Email: o.mwabonje@gmail.com,JiaQian.Jiang@gcu.ac.uk
}

\begin{abstract}
The purpose of this paper is to explore the use of solvent extraction for the removal and recovering phosphate from wastewater and water sources. The results revealed that to achieve the maximum phosphate removal, the best extractant was a mixture of kerosene and benzyldimethylamine at a volume ratio of 2:1. A phosphate extraction efficiency of greater than $80 \%$ was achieved on the wastewater samples tested; a model solution and real sewage. A high stripping efficiency of greater than $90 \%$ was achieved from stripping, using $6 \mathrm{M}$ sulphuric acid. By mixing the recycled to fresh extractants at volume ratios of 2:1, it was possible to re-use the resulting extractant from the stripping process nine times, while maintaining the overall phosphate recovery efficiency. This research revealed that solvent extraction is feasible in the respect of phosphate removal and recovery and has potential for use as an alternative method for industrial applications.
\end{abstract}

Keywords: Extractant, Phosphorus Removal, Solvent Extraction (SE)

\section{Introduction}

Eutrophication is accelerated due to the increase in the phosphorus and nitrogen loading in water and the increased growth of undesirable algae and aquatic weeds as a result of the over loading of phosphorus [1]. Due to this, water utility for fisheries, recreation, industry and drinking gets restricted [2]. A periodic surface bloom of cyanobacteria (blue-green algae) may appear in drinking water supplies and could be hazardous to both animals and human beings. An increased awareness on eutrophication and the need for solutions has been attributed to neurological damage in people due to exposure to highly toxic and volatile chemicals produced by the algae [3].

A greater interest in solvent extraction (SE) for large-scale industrial application was initiated by the production of uranium and the reprocessing of irradiated nuclear materials in the U.S. Manhattan project [4]. This aroused greater interest in the use of SE in other industries for the separation and purification of their products. Examples of this approach are recovering metals from water treatment sludge and effluents [5,6]. SE has also been utilised in the remediation of contaminated soils. The pollutants are transferred to and carried off by the liquid phase. The soil and the extracting agent are separated once the pollutants are transferred to the fluid phase. Where feasible and economical, the fluid phase is treated and then recycled. The selection of an extracting agent has to take into account safety both to humans and the environment [7].

In solvent extraction, a small quantity of an organic soluble chemical called the extractant is dissolved in a second organic liquid called the diluent. The mixture is often referred to as the solvent or the organic phase. The diluent may be a material such as kerosene or similar hydrocarbon. During the extraction process, the extractant reacts chemically with the phosphates in the aqueous phase forming a phosphate-extractant complex that is soluble in the kerosene [8]. Generally, SE takes place at ambient pressures and the liquid range extends from about freezing up to about boiling temperature [9]. In this study, benzyldimethylamine a cationic surfactant has been employed as an extractant. These types of surfactants are those which the ionic group on the hydrophobic have a positive charge. Most of the available cationic surfactants are based on a nitrogen atom carrying a positive charge while in others the positive charge is carried by phosphorus or a sulphur atom [10]. 
On the other hand, in view of the limited resources of phosphorus and the increasing concern of sustainability, the recovery of phosphorus from wastewater is a promising and laudable approach [11,12]. The aim of this study was to remove and recover phosphates in water and wastewater using the LLE approach. This was to be achieved by selecting optimum diluents and extractants and there mixing ratios (between wastewater diluent and extractants). Similarly, the strength and length of mixing and phase separation were to be investigated.

\section{Materials and Methodology}

\subsection{Phosphorus Analysis Method}

The ascorbic acid method [13] was used for phosphorus analysis. In principal, ammonium molybdate and potassium antimonyl tartrate react in acid medium with orthophosphate to form a heteropoly acid (phosphomolybdic acid) that is reduced to intensely coloured molybdenum blue by ascorbic acid. The minimum detection limit for this method is $10 \mu \mathrm{g} / \mathrm{L}$.

\subsection{Laboratory Set-Up}

A batch process was used for both extraction and stripping experiments. The extractant and the samples were mixed together in duran bottles and the contents thoroughly shaken using a shaker. The mixture was then transferred to separate in separating funnels.

\subsection{Surfactant and Diluents Selection}

Seven different cationic surfactants were selected and subjected to solubility tests on both water and diluents (kerosene, acetone and ethanol). The cationic surfactants that were selected and investigated upon are namely Benzalkonium chloride, Benzyldimethylamine, Dibenzylamine, 2 ethyl-4-methylimidazole, Hexadecyltrimethylammoniumbromide, Dodecyltrimethylammonium bromide and Tritylamine.

\subsection{Model Water Preparation}

The model water was prepared using a commercial phosphate standard solution $1000 \mathrm{mg} / \mathrm{L}$ [14]. The concentration was made in the range of 1 and $30 \mathrm{mg} / \mathrm{L}$ by taking different volumes from the stock solution and filling to the mark in $250 \mathrm{ml}$ volumetric flasks with distilled water.

\subsection{Wastewater Sampling}

The wastewater sample used in this study was obtained from Southern Water in Brighton UK. The sample was taken after the pre-sedimentation stage and its characteristics determined in the lab. The results are as shown on the Table 1 below.

\subsection{Model and Wastewater Extraction Study}

Solubility tests: $10 \mathrm{~mL}$ of an extractant was placed into a graduated conical flask. A similar volume of water was then added and the flask covered with a stopper. The mixture was then shaken thoroughly using a shaker (KS125, Merck Ltd, England) at a speed of $250 \mathrm{rpm}$ for 10 minutes. As soon as the agitation time had elapsed, the conical flasks were left to stand for 30 minutes in a fume cupboard. Observations were then recorded on the miscibility of the extractant to water. The same procedure was then followed in selecting a suitable diluent this time substituting water in the mixture for a diluent.

Kinetics of extraction (mixing and separation time): In order to determine the best mixing time, a volume $(\mathrm{mL})$ of the extractant (benzyldimethylamine + kerosene) and model water containing known phosphate concentration, at a phase ratio of 1:1 was placed in duran bottles (500 $\mathrm{mL})$. The concentration levels of the model water varied from 1 to $30 \mathrm{mg} / \mathrm{L}$. The mixtures were then shaken in a shaker (KS125, Merck Ltd, England) at a speed of 250 rpm at a time interval ranging from 1 to 24 hours. The contents were then transferred into separating funnels (500 $\mathrm{mL}$ ) and aqueous phase tested for the remaining phosphate concentration. The same procedure was used to determine the best separation time by varying the amount of time by which the phases separated in the separating funnels between 2 and 24 hours.

Extractant/diluent phase ratio: The best extractant/diluent phase ratio was determined using the phase variation method. The mixture of the surfactant and kerosene at different ratios formed the organic phase used in the extraction process. This was varied in order to optimise the operating conditions. The organic phase (Kerosene and Benzyldimethylamine) was prepared using the ratios $1: 1,1: 2,2: 1,1: 4,4: 1,6: 1,9: 1,15: 1$ and $20: 1$. The organic phase was then placed in duran bottles $(500 \mathrm{~mL})$ and model water added at a volume ratio of 1:1. The mixtures were thoroughly shaken using a shaker (KS125, Merck Ltd, England) for 6 hours at a speed of

Table 1. Wastewater quality parameters.

\begin{tabular}{lc}
\hline Quality Parameters & Values \\
\hline $\mathrm{pH}$ & 7.36 \\
Suspended Solids (mg/L) & 36 \\
Total P (mg/L) & 22.86 \\
Soluble P (mg/L) & 21.27 \\
Turbidity (NTU) & 52.2 \\
\hline
\end{tabular}


$250 \mathrm{rpm}$. The phases were then allowed to separate in separating funnels $(250 \mathrm{~mL})$ for 2 hours. The organic phase of the varied phase ratios was used to extract phosphates from model water of a wider range of $\mathrm{P}$ concentration (1 to $30 \mathrm{mg} / \mathrm{L}$ ).

\subsection{Model and Wastewater Stripping Study}

Separation time: In order to determine the separation time, the organic phase obtained from the extraction process was mixed with a stripping agent at a volume ratio of $1: 1$. This comprised of $125 \mathrm{~mL}$ of organic phase mixed with a similar amount of the stripping agent. The organic phases used were those that came from the extraction involving model water samples with starting phosphorus concentration of 3, 6 and $10 \mathrm{mg} / \mathrm{L}$ respectively. The mixture was then placed in duran bottles (500 $\mathrm{mL}$ ) and shaken in a shaker at a speed of $250 \mathrm{rpm}$ for 4 hours. The mixture was then transferred into separating funnels and allowed to separate for time intervals varying between 1 to 24 hours.

Organic-P/acid phase ratio: The stripping agents chosen for the study were sulphuric and hydrochloric acid. The organic phase from the extraction step was mixed with the acid at $1: 1,1: 2,2: 1,1: 4$, and $4: 1$ ratio so as to determine the best phase ratio. The two solutions were placed in duran bottles (500 $\mathrm{mL}$ ) and the mixture thoroughly shaken using a shaker (KS125, Merck Ltd, England) for 4 hours at a speed of $250 \mathrm{rpm}$. In this particular experiment, the organic phases used were those taken from the extraction of aqueous solutions with starting phosphorus concentration of 3, 6 and $10 \mathrm{mg} / \mathrm{L}$ respectively. The phases were then allowed to separate in separating funnels $(250 \mathrm{ml})$. Different quantities of phosphates were to have been transferred from the organic phase to the sulphuric acid phase. The phosphate recovered was determined by examining its concentration in the acid phase using the ascorbic acid method.

\subsection{Extractant Recycling Study}

The organic phase obtained after the stripping process was re-used again in the extraction work in order to study the extraction efficiency of such a reagent for long term use. Three kinds of ratio between the recycled and fresh organic phases were investigated in the same way as the experiment in the lake water study. The fresh phase was composed of a mixture of kerosene and surfactant at a ratio of $2: 1$. The ratios of the fresh to recycled extractants were 1:1, 1:2, 1:4. The different diluents formed as a result were mixed with the sewage samples in Duran bottles $(250 \mathrm{~mL})$ at the phase ratio of $1: 1$ and the mixture thoroughly shaken using a shaker (KS125, Merck Ltd, England) for $6 \mathrm{hrs}$ at a speed of $250 \mathrm{rpm}$ and the mixture allowed to separate in separating funnels for 2 hours. Different quantities of phosphates were to have been transferred from the aqueous phase to the organic phase. The organic phase was then collected and stripped using sulphuric acid (6M) following the same stripping procedure as outlined above. The same acid used for stripping the organic phase of the fresh extractant was reused in the extractant recycling study in order to minimise the amount of the acid used. The experiment was conducted for eight runs with each run involving a reuse of the extractant with an addition of a freshly prepared one at the same ratio of 1:2. The aqueous phase in each subsequent run was analysed for the remaining phosphates using the ascorbic acid method.

\section{Results}

\subsection{Model Water Study}

In order to separate and concentrate phosphates in the acid extract, an investigation was carried out on the efficiency of solvent extraction using several cationic surfactants with respect to the procedure outlined in above. The preliminary experiments based on solubility tests of the seven chosen cationic surfactants (Table 2) revealed that benzyldimethylamine and dibenzylamine could be used as extractant as they were to be miscible in kerosene (diluent) but immiscible in water. However, benzyldimethylamine was the most effective extractant to

Table 2. Solubility of chosen surfactants in selected diluents.

\begin{tabular}{|c|c|c|c|c|}
\hline \multirow{2}{*}{ Surfactant } & \multicolumn{4}{|c|}{ Diluent } \\
\hline & Kerosene & Acetone & ethanol & Water \\
\hline Benzalkonium chloride & Insoluble & Soluble & Soluble & Soluble \\
\hline Benzyldimethylamine & Soluble & Soluble & Soluble & Insoluble \\
\hline Dibenzylamine & Soluble & Soluble & Soluble & Sparingly soluble \\
\hline 2 ethyl-4-methylimidazole & Soluble & Soluble & Soluble & Soluble \\
\hline Dodecyltrimethylammonium bromide & Insoluble & Soluble & Soluble & Soluble \\
\hline Tritylamine & Soluble & Soluble & Insoluble & Soluble \\
\hline
\end{tabular}


increase the extraction efficiency of phosphates to the solvent phase and decreasing that of the extractant to aqueous phase in comparison to dibenzylamine using kerosene as diluent.

In addition, benzyldimethylamine comes in liquid form from the manufacturer hence making it easy to use. In comparison with the other surfactants selected for this study, benzyldimethylamine was found to be relatively cheap to purchase, therefore it was selected for all the subsequent work.

The effect of mixing time on extraction was investigated. The time of mixing was varied between $1 \mathrm{hr}$ to 24 hrs. Three levels of starting P concentrations namely 3, 6 and $10 \mathrm{mg} / \mathrm{L}$ were considered for this experiment. The results show that the extraction efficiency initially remains constant but starts to decrease after 8 hrs of agitation. This shows that although diffusion is influenced by the stirring process, it has very little impact on extraction. The mixing time was therefore fixed at 6 hrs for all other experiments since high extraction efficiency was realised at this level with respect to all the initial phosphates concentration considered.

Nevertheless, the study also demonstrates that the volume ratio of kerosene to surfactant is an essential parameter which affects the extraction efficiency and indeed the entire treatment cost. The optimum volume ratio of kerosene to surfactant was 2:1 based on the extraction efficiency (Figure 1). The extraction efficiency under this volume ratio was greater than $80 \%$ for the starting phosphates concentration of greater $3 \mathrm{mg} / \mathrm{L}$.

Similarly, sets of experiments were conducted in order to determine the effects of separation time on the extraction efficiency. In order to prepare the extractant for the experiment, kerosene was mixed with benzyldimethylamine at a ratio of 2:1. The separation time was varied from 2 hrs to 24 hrs while the range of starting P concentration varied between 1 to $30 \mathrm{mg} / \mathrm{L}$. The results are represented in Figure 2. The difference in extraction efficiency between various separation hours was insignificant. Therefore, it was agreed that 2 hours separation time was adequate for achieving practical extraction efficiency.

Meanwhile, sulphuric and hydrochloric acids were used in the stripping process. Three levels of the acid concentrations were investigated for their stripping efficiency on the phosphate rich organic phase obtained after the extraction process. These included 3M, 6M and 9M for the case of sulphuric acid and $2 \mathrm{M}, 4 \mathrm{M}$ and $6 \mathrm{M}$ for hydrochloric acid respectively. The results are represented in Figure 3. The results obtained did not register significant differences in terms of acid type and concentration used and a stripping efficiency of $>90 \%$ was achieved irrespective of the acid type and concentration
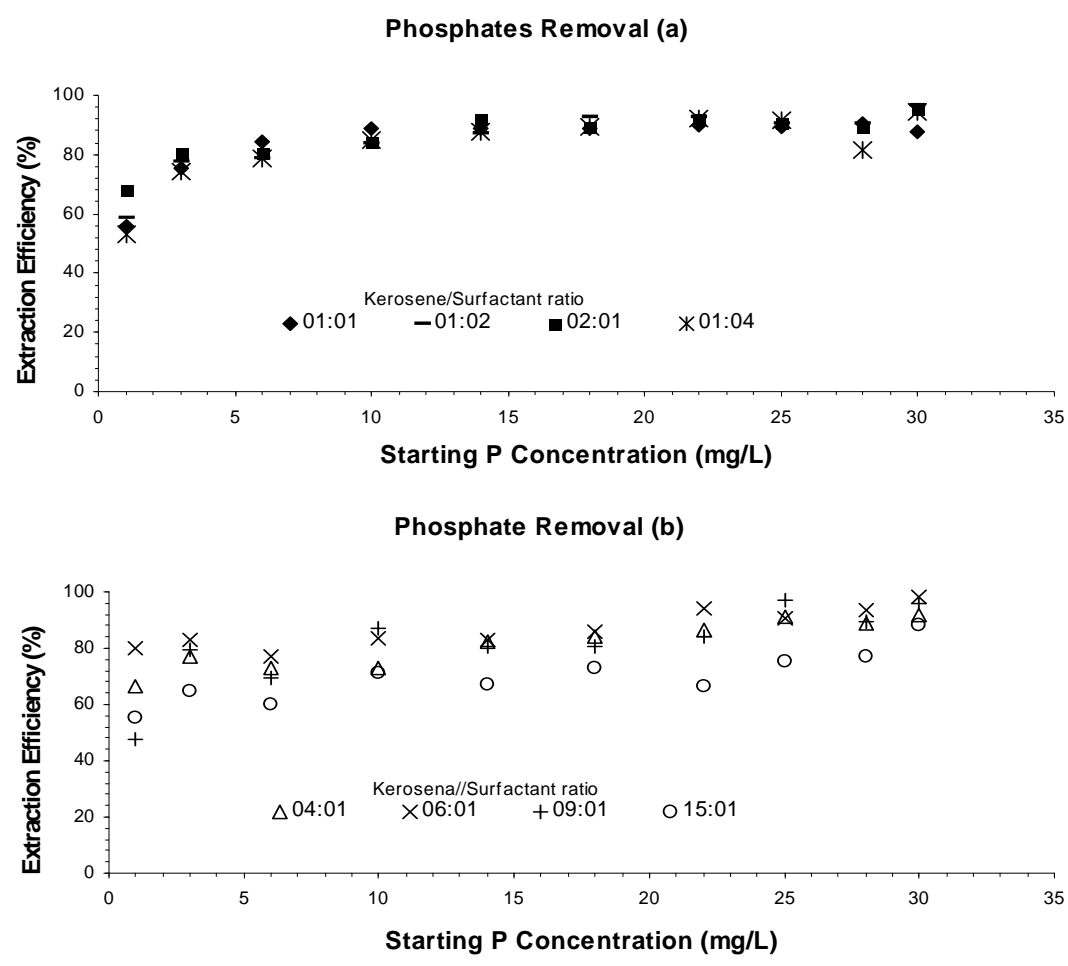

Figure 1. Comparison of different Kerosene and Benzyldimethylamine Ratios used in Extraction of Phosphates in Relation to Extraction Efficiency. The results are presented as (a) and (b) for eight kerosene / Benzyldimethylamine ratios. 

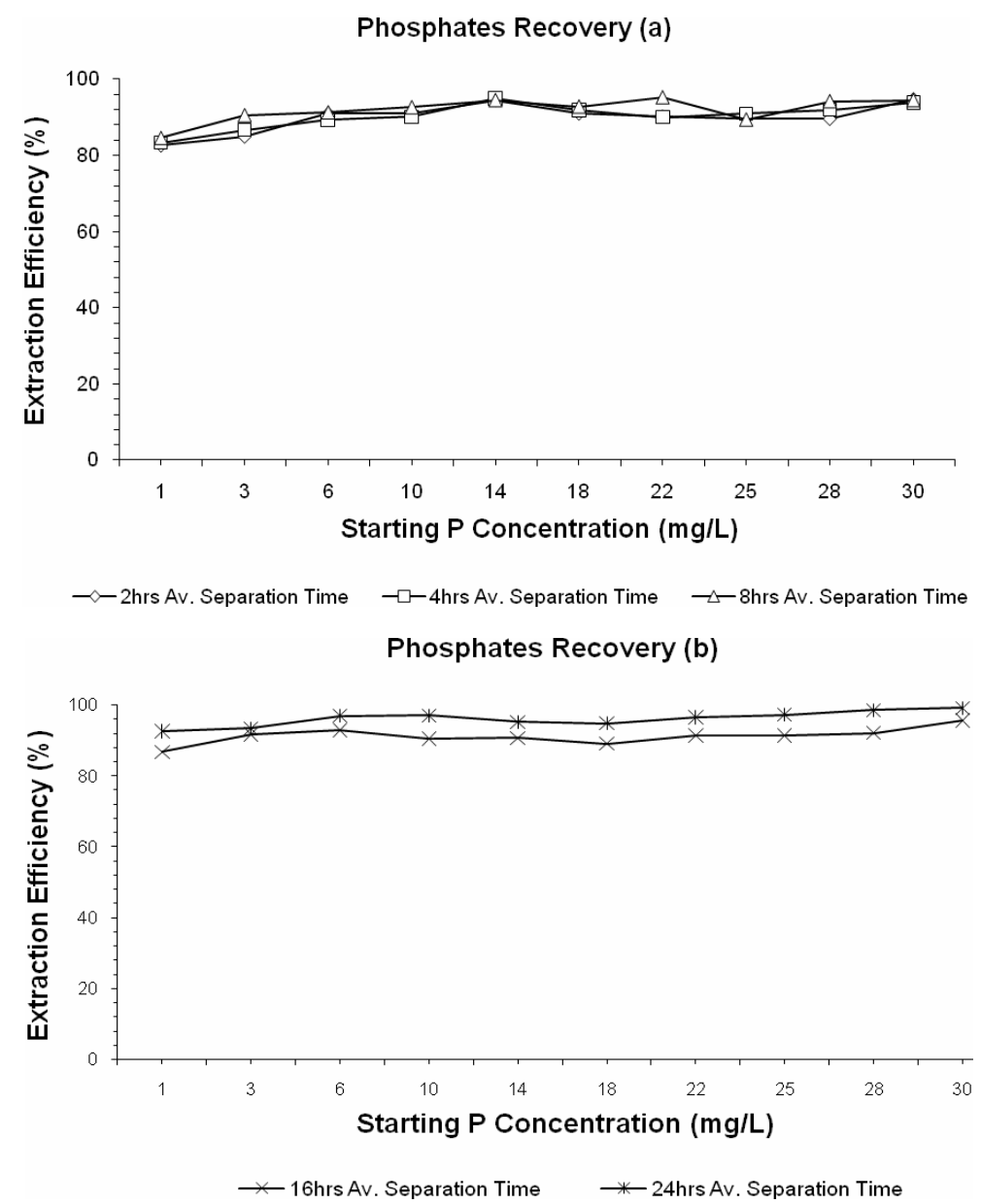

Figure 2. The effect of separation time on the extraction of Phosphates. The results are presented as (a) and (b) for five separation times.

(Figure 3). Five kinds of mixing ratios (1:1, 1:2, 1:4, 2:1, and $4: 1$ ) between the organic phosphate rich phase and the stripping media were also investigated as evident in Figure 3. The results demonstrated an increase in stripping efficiency with increase in the volume of the acid. A 1:1 ratio by volume was accepted as adequate to achieving practical stripping efficiency at an agitation period of 4 hours. Since the studies on the two acids did not show significant differences, sulphuric acid (6M) was selected as the main stripping agent and therefore used in subsequent experiments. Table 3 summarises the optimal operating conditions of SE of phosphate.

\subsection{Wastewater Study}

Using the established extraction and stripping conditions, the SE efficiency was examined for real wastewater samples, which were collected from a full-scale wastewater treatment works from Southern Water Limited in England. The quality composition of the wastewater was analysed as shown in Table $\mathbf{1}$ above. The study demon- strates that in spite of the high amount of suspended solids in the wastewater samples, an overall extraction efficiency of greater than $86 \%$ (Table 4) was achieved using

Table 3. A summary of extraction conditions established during model water studies

\begin{tabular}{|c|c|c|c|}
\hline \multicolumn{2}{|l|}{ Extraction Parameter } & \multicolumn{2}{|c|}{ Stripping Parameter } \\
\hline Best Surfactant & $\begin{array}{l}\text { Benzyldi- } \\
\text { methyl-amine }\end{array}$ & Stripping Agent & $\begin{array}{l}\mathrm{H}_{2} \mathrm{SO}_{4} \\
(6 \mathrm{M})\end{array}$ \\
\hline Best Mixing time & $6 \mathrm{hrs}$ & Mixing time & 4 hrs \\
\hline $\begin{array}{l}\text { Best separation } \\
\text { time }\end{array}$ & $2 \mathrm{hrs}$ & $\begin{array}{l}\text { Best separation } \\
\text { time }\end{array}$ & $2 \mathrm{hrs}$ \\
\hline $\begin{array}{l}\text { Mixing Ratio } \\
\text { (Kerosene: Sur- } \\
\text { factant) }\end{array}$ & $2: 1$ & & \\
\hline $\begin{array}{l}\text { Mixing Ratio } \\
\text { (aqueous: Organic } \\
\text { Phase) }\end{array}$ & $4: 1$ & $\begin{array}{l}\text { Mixing Ratios } \\
\text { (Organic: Acid } \\
\text { Phase) }\end{array}$ & $1: 1$ \\
\hline $\begin{array}{l}\text { Minimum detection } \\
\text { limit }\end{array}$ & $10 \mu g / L$ & $\begin{array}{l}\text { Minimum detec- } \\
\text { tion limit }\end{array}$ & $10 \mu g / L$ \\
\hline $\begin{array}{l}\text { Maximum extrac- } \\
\text { tion efficiency }\end{array}$ & $95.60 \%$ & $\begin{array}{l}\text { Maximum strip- } \\
\text { ping efficiency }\end{array}$ & $91.34 \%$ \\
\hline
\end{tabular}


Table 4. Wastewater phosphorus extraction results.

\begin{tabular}{lllll}
\hline & $\begin{array}{l}\text { Fresh } \\
\end{array}$ & $\begin{array}{l}\text { Fresh: } \\
\text { Organic }\end{array}$ & Recycled extrac- \\
\cline { 3 - 5 } & Phase & $1: 1$ & $1: 2$ & $1: 4$ \\
\hline Extraction Efficiency (\%) & 87.00 & 81.88 & 71.35 & 62.54 \\
& 86.82 & 81.84 & 78.63 & 61.50 \\
$\begin{array}{l}\text { Average Extraction Effi- } \\
\text { ciency (\%) }\end{array}$ & 86.91 & 81.86 & 74.99 & 62.02 \\
\hline
\end{tabular}

a freshly prepared extractant. Three ratios of fresh to recycled extractants (1:1, 1:2 and 1:4) were investigated as illustrated in Table 4. There was a general decrease in the extraction efficiency with respect to the increase in the recycled portion of the extractant. This implies that the introduction of the fresh extractant enhanced the extraction of phosphates from the wastewater. The 1:2 ratio of fresh to recycled extractant was found to be the most reasonable ratio for separation and concentrating phosphates efficiently from wastewater since a 1:4 ratio gave low extraction efficiency. In addition, this selected ratio does emphasise the aim of sustainability and cost reduction by using more of the recycled extractant while maximising extraction efficiency. The stripping experi- ments conducted using $6 \mathrm{M}$ sulphuric acid achieved an average stripping efficiency of $94 \%$.

The study also demonstrates that the extractant has the capability of being recycled nine times before its efficiency falls to an average of $65 \%$ for total residual $\mathrm{P}$ concentration and $70 \%$ for soluble residual $\mathrm{P}$ concentration, one example is illustrated in Figure 4. The experiments help to show that the extracted phosphates can be recovered in the form of an acid which can be utilised for other industrial uses including fertiliser production.

\section{Discussion}

There are several approaches used to extract phosphates from wastewater. One approach is to specifically design an extractant as a cationic host with the ability to bond reversibly with the guest and in this study the guest being the phosphate anion. The cations can have metal centres that bind to the phosphates anion group or cationic group that interact by hydrogen bonding or donating an electron. Amines meet many of these requirements and reversibility of the anionic binding can be achieved by switching between the free amines and its protonated

Phosphates Recovery (a)

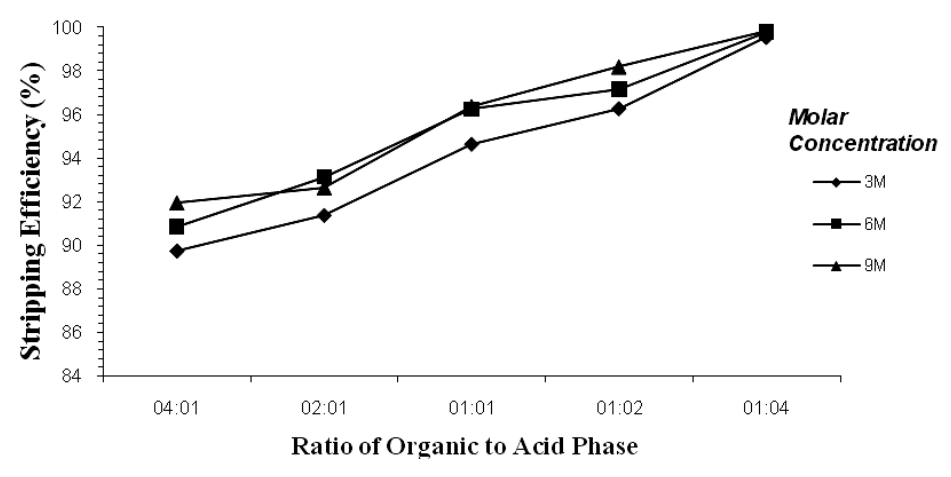

Phosphates Recovery (b)

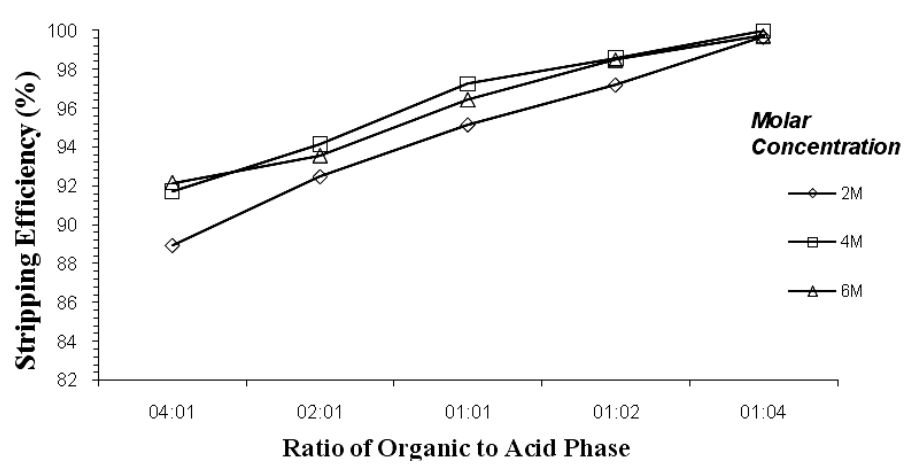

Figure 3. The effect of organic to acid phase ratios on the stripping process of phosphates using (a) sulphuric acid and (b) hydrochloric acid. 


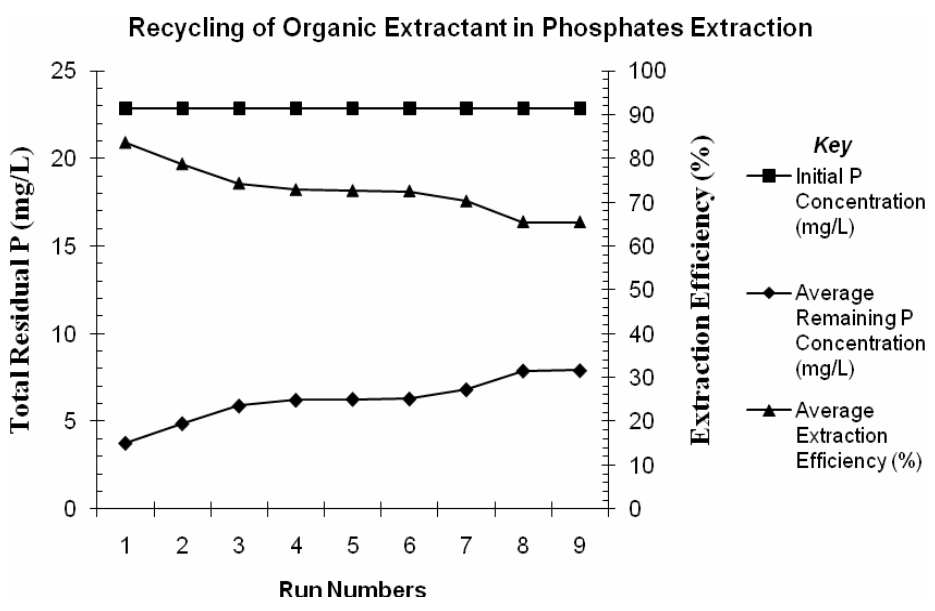

Figure 4. Relationship between the extraction efficiency, total phosphate residual with respect to the experimental run numbers. The ratio of fresh to recycled extractant was maintained as 1:2.

form. In this research study the surfactant (benzyldimethylamine), an amine reagent, was used as a cationic host or extractant, together with kerosene as a diluent containing an amine group. Selection of an appropriate diluent is very important due to both physical and chemical effects that they can exhibit in the extraction process and also the economics of the entire system. Kerosene was selected in this research work due to its inert properties and as it had been utilised as a diluent in many previous liquid-liquid extraction studies. Kerosene is also relatively cheap to purchase and readily available. Kerosene aided by decreasing emulsion formation during agitation, a phenomenon common with surface active extractants such as benzyldimethylamine.

Amines are nucleophiles and can act as Bonsted-Lowry acids by donating a proton. The results evidently demonstrate that the phosphate ions can be selectively and reversibly bound on the extractant, permitting their subsequent removal from the wastewater effluent. The extractant also displays excellent selectivity of phosphates. This could be attributed to the higher valence (ionic charge) of the phosphates anions in solution. The extractant, composed of kerosene and Benzyldimethylamine, when added to wastewater can form micelles which have positive charge to their surface. Anionic contaminants, such as phosphate, which is the focus of this research work, can be bound on these micelles by electrostatic interaction. This then enables the micelle-pollutants complex to be available for removal from the wastewater effluent.

The experimental results, obtained via batch experimentation using wastewater samples which contained varying amounts of phosphate, demonstrated a $>90 \%$ binding capacity of the extractant. This could be attributed to the higher anion concentration gradient between the surrounding liquid medium and the "phosphate free" extractant matrix, which increases the flux of phosphate anions into the cationic extractant matrix to provide a higher binding capacity. Similarly, the extractant has shown a substantial capability to extract phosphate anions across a wide concentration range, as indicated by the results obtained for each of the sample categories considered in this research.

The stripping process can be described as the reverse reaction to the extraction process. Thus, in this research work, an acid solution has been used of a concentration sufficiently high to drive the phosphate ions from the organic phase into the acid phase. It therefore follows, from the stripping results illustrated above that the stability of the extracted species does govern the type and solution of the strip and concentration required. Hence a high concentration of acid $\left(6 \mathrm{M} \mathrm{H}_{2} \mathrm{SO}_{4}\right)$ is needed to strip phosphates from the organic phase in the stripping stage. Consequently, the extractant can be regenerated after having bound the phosphate ions from the wastewater and its removal efficiency can be retained for at least five regeneration cycles upon treatment with 6M sulphuric acid. Most aliphatic amines that are used in liquid-liquid extraction operations have low solubility in aqueous acidic solutions as low as $10 \mathrm{ppm}$ [15]. This solubility is determined, amongst other things, by the number of carbon atoms in the molecule and the degree of chain branching, as the greater the branching the lower the solubility. As demonstrated by the stripping results in this research work, it follows that the extractant used has a lower solubility in the $6 \mathrm{M} \mathrm{H}_{2} \mathrm{SO}_{4}$ used as the stripping agent. Nevertheless, by adding a small percentage of a fresh extractant to the stripped volume enabled it to be reused over multiple cycles for the removal of phosphates from wastewater. The sulphuric acid used for 
stripping the organic phase from the wastewater demonstrated that it could be reused nine times in a similar manner to the extractant.

$\mathrm{SE}$ is an equilibrium process; however, the rate at which the equilibrium is reached is an essential factor. In SE processing, the kinetics of the systems governs the throughput of the process. Accordingly, with slow kinetics, the retention time in the extraction stages must be greater than for a system involving fast kinetics. Indeed very fast kinetics of extraction allows the use of contactors which have retention times in the order of seconds [15]. Normally, the extraction is governed by mass transfer and diffusion rates which can be relatively rapid. The viscosity of the phases, the amount of agitation and the temperature of the system are some of the other factors that affect the rate of extraction. In a heterogeneous system, such as we have in SE, the rate of extraction depends in large measure on the surface area of the dispersed phase. In using the shaker to mix the organic and the aqueous phase, one of the phases gets dispersed into the other and the amount of the surface area of the dispersed phase largely depends on the amount of agitation. However, it is not necessarily that the greater the agitation the greater the rate of phosphate ion extraction. In this study, the agitation was done at a fixed rate of 250 rpm which was sufficient enough to allow extraction to take effect. Too much agitation can result in the formation of stable or semi-stable emulsions.

Moreover, decreasing the bubble size of the dispersed phase could result in making the bubbles resemble rigid spheres. In this situation, there is no internal movement within the spheres, hence no new surfaces are produced and the extractant cannot get to the surface to react with the phosphate ions, resulting into a slow extraction rate. The desorption of surfactants used as extractants such as amines (benzyldimethylamine in this study) from the organic-aqueous interface, is a rate determining step. This effect reduces the rate of transfer of anionic/cationic species as a result of mechanical blocking, especially if the interfacial area is large due to excessive mixing [15]. Thus extraction with excessive mixing may be significantly different from that obtained from gentle mixing with the same system, as equilibrium is not attained in the former case. Again, using a low mixing rate of 250 rpm provided the right equilibrium by which the transfer of phosphate ions from the aqueous phase into the organic phase was achieved and the same scenario repeated at the stripping stage. This helps to explain that increasing the energy of mixing does not necessarily mean that the rate of extraction will also increase.

The physical aspects of SE are largely concerned with the dispersion of the two phases on mixing and the completeness of the phase disengagement or coalescence.
The two processes, i.e., dispersion and coalescence, are integral steps in the SE process and indeed govern the design and operation of a plant. Therefore, in order to achieve a practically useful level of coalescence of two dispersed phases, the dispersion must be of a temporary nature [15]. The rate of mass transfer across a phase boundary is a function of the drop size distribution or the interfacial area between the phases. The drop size is controlled by the speed and the type of the shaking device and also the surface tension and the densities of the two phases. It follows then that the smaller the drop size, the greater the rate of mass transfer; hence the agitation of the two phases has an impact on the mass transfer rate of the phosphate ions and the coalescence of the dispersed phase. Meanwhile, the high extraction and stripping efficiency realised in this research indicates the high mass transfer rates in between phases, meaning between the aqueous/organic phase and organic/acid phases. The extractant has demonstrated to have the capability of selectively binding phosphorus into its matrix, permitting its subsequent removal from the contaminated source of wastewater. The extractant containing the bound phosphorus can then be regenerated for reuse. The treated effluent can then be safely discharged into natural waters or sent for further treatment for use as a municipal water supply. The phosphoric acid produced by stripping the extractant with sulphuric acid can be used as a raw material in the production of fertiliser among other industrial uses.

\section{Conclusions}

This study revealed that by controlling certain parameters such as equilibrium time (6 hours and 4 hours for extraction and stripping, respectively), benzyldimethylamine/kerosene ratio of $1: 2$ by volume and using $6 \mathrm{M}$ $\mathrm{H}_{2} \mathrm{SO}_{4}$ as a stripping reagent, a high extraction efficiency of between $80-90 \%$ and a stripping efficiency of $>90 \%$ can be achieved.

In the wastewater study, an overall extraction efficiency of $87 \%$ efficiency was achieved. A ratio of 1:2 of the fresh to the recycled extractant was preferred, as it gave a reasonable extraction efficiency of $>80 \%$. The stripping efficiency was 94\%. Meanwhile, the resulting extractant was able to be reused nine times, achieving an overall phosphate removal efficiency of between 71$91 \%$.

\section{References}

[1] EA, “Aquatic Eutrophication in England and Wales: Proposed Management Strategy,” Environmental Agency Environmental Issue Series, Bristol, UK, 2003. 
[2] S. Burk, L. Heathwaite and N. Preedy, "Transfer of Phosphorus to Surface Waters; Eutrophication,” In: Phosphorus in Environmental Technologies Principles and Applications, IWA Publishing, London, 2004, pp. 120-140.

[3] A. N. Sharpley, T. Daniel, T. Sims, et al. "Agricultural Phosphorus and Eutrophication,” ARS-149, US Department of Agriculture (USDA)-Agriculture Research Service (ARS), University Park, PA, 1999, pp. 37.

[4] M. Cox and J. Rydberg, "Introduction to Solvent Extraction in Solvent Extraction Principles and Practice," Marcel Dekker, Inc, New York, 2004.

[5] J. Q. Jiang, "Recovery and Reuse of Aluminium Coagulants from Coagulation Sludge by Liquid-Ion Exchange,” In: H. H. Hahn, E. Hoffmann and H. Odegaard, Ed., Chemical Water and Wastewater Treatment VI, SpringerVerlag, Berlin, 2000, pp. 373-382.

[6] J. Q. Jiang, A. Panagoulopoulos and Y. Zhang, "Recovery and Re-Use of $\mathrm{Al}$ and Fe Based Coagulants from Coagulation Sludge,” In: J. Miles, D. Smith, P. Owen, Ed., Management of Wastes from Drinking Water Treatment, CIWEM, London, 2002, pp. 181-189.

[7] A. M. O. Mohamed and H. E. Antia, "Developments in Geotechnical Engineering, 82-Geoenvironmental Engineering," Edition 1, Elsevier Science Ltd., Amsterdam, 1998.

[8] D. A. Cornwell, "An Overview of Liquid Ion Exchange with Emphasis on Alum Recovery,” Journal of the American Water Works Association, Vol. 71, No. 12, 1979, pp. 741-745.

[9] Y. Marcus, "Principles of Solubility and Solution,” In: J. Rydberg, C. Musikas and G. R. Choppin, Ed., Principles and Practices of Solvent Extraction, Mercel Dekker, Inc, New York, 1992.

[10] W. S. Perkins, "Surfactants-A Primer, an Indepth Discussion of the Behaviour of Common Types of Surfactants," ATI-Dyeing, Printing and Finishing, 1998, pp. 51-53.

[11] C. Schaum, P. Cornel and N. Jardin, "Possibilities for a Phosphorus Recovery from Sewage Sludge Ash,” In: Proceedings of the IWA Specialist Conference on Management of Residues Emanating from Water and Wastewater Treatment, Johannesburg, South Africa, 2005, pp. 9-12.

[12] American Water Works Association, "Standard Methods for the Examination of Water and Wastewater," 19th edition, American Water Works Association, Washington DC, 1995.

[13] VWR, "Phosphate Standard Solution,” 2003. http:// uk.vwr.com/app/Home

[14] G. M. Ritcey and A. W. Ashbrook, "Solvent Extraction Principles and Applications to Process Metallurgy Part II,” Elsevier Scientific Publishing Company, Chaps 4-5, New York, 1979. 